\title{
Generating architectural plan with evolutionary multiobjective optimization algorithms: a benchmark case with an existent construction system
}

\author{
Giuseppe Canestrino \\ Department of Civil Engineering, University of Calabria | Italy | giuseppe.canestrino@unical.it \\ Greco Laura \\ Department of Civil Engineering, University of Calabria | Italy | laura.greco@unical.it \\ Francesco Spada \\ Department of Civil Engineering, University of Calabria | Italy | francesco.spada@unical.it \\ Roberta Lucente \\ Department of Civil Engineering, University of Calabria | Italy | roberta.lucente@unical.it
}

\begin{abstract}
In architectural design, evolutionary multiobjective optimization algorithms (EMOA) have found use in numerous practical applications in which qualitative and quantitative aspects can be transformed into fitness functions to be optimized. This paper shows that they can be used in an architectural plan design process that starts from a more traditional approach. The benchmark case uses a novel construction system, called Ac.Ca. Building, with a vast architectural and technological database, arleady validated, to generate architectural plan for a residential towerbuilding with a parametric approach and EMOA. The proposed framework differs from past research because uses spatial units with high level of architectural and tecnological definition.
\end{abstract}

Keywords: Architectural design; Parametric architecture; Performance-driven design; architectural layout; evolutionary multiobjective optimization.

\section{INTRODUCTION}

This paper shows that computational and parametric design tools can be implemented in the use of an arleady designed construcion system characterized by an high level of architectural and technological definition. With these tools, optimized solutions to architectural design problems can be developed making the best use of a construction system.

A benchmark case concerning the generative design of highly prefabricated architectural plan is presented. The spatial units used have already been designed with respect to the minimum requirements for residential use in the Italian context. The proposed framework is to be used in the preliminary stage. Therefore, the generative algorithm was developed according to the principles of agile coding (Beck, et al., 2001). The search for the most promising solutions is done through the use of EMOA.

The Ac.Ca. Building construction system is presented and used in this paper. Several features of spatial flexibility makes this system suitable for a computational approach in space layout generation. Different approaches used for the generation of spatial layout are presented to draw analogies with the proposed framework. Afterwards the used framework and generative algorithm are presented with attenction to theoretical and pratical aspects encountered in the coding phase. In particular, the design of the spatial layout of a small residential tower presented in this paper.

It is specified that the developed algorithm isn't a generalized approach for the design of architectural layouts, but is strictly linked to the use of the Ac.Ca. Building construction

\section{AC.CA. BUILDING CONSTRUCTION SYSTEM}

The prosed contribution (Section Architecture; Research \& Experimentation) presented in this paper concerns the research and development project "Ac.Ca. Building. Design and build in safety with steel and hemp. Innovative technologies for sustainable buildings" where Ac.Ca. stands for Acciaio - Canapa. The project is currently undergoing prototyping on a 1:1 scale. The initiative, financed within the POR Calabria FESR- 2014-2020, is based on a partnership that engages the University of Calabria and companies present on international markets The partnership aims to involve SMEs in the production of dry industrialized systems in a context characterized by the hegemonic presence of concrete. The research project is still under development but several results are accessible and published by Roberta Lucente (2020).

\section{AC.CA. BUILDING MAIN OBJECTIVES AND BACKGROUND}

Ac.Ca. Building is an integrated system of typological and technological solutions with high flexibility for the residence (collective, co-housing, mixed systems) and for advanced working spaces (co-working offices, fab-lab). It was developed for the needs of users on the move for necessity and / or work and to participate in the formation of useful tools for the policies of public and private promoters for the construction and management of new construction products. 


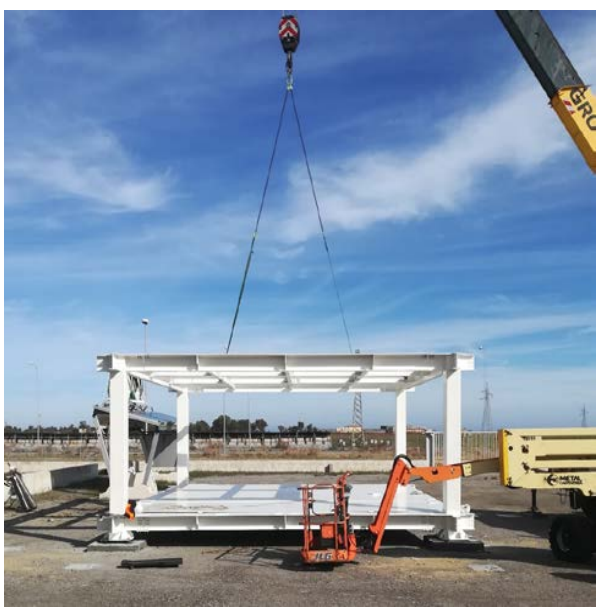

Figure 1: prototyping of the Ac.Ca. Building construction system. Assembly of the main structure

The proposal takes into account a cultural framework characterized by the extension of the concept of temporariness as an ordinary socio-economic structure. This led to use quality profiles typical of permanent buildings and attention to spatial and technological flexibility in social housing in the proposed construction system. Also a renewed interested on the sustainability of industrialized building led to face face the obsoloscence of spaces and technologies.

Ac.Ca. building, through focusing on flexible and adaptable typlogical schemes, is characterized by the enhancement of the use of steel in the residence. It defines an open construction system (with recyclable and customizable industrialized components in a logic of mass customization process) that combines the steel frame with closures and partitions made mainly with hemp fiber products, hitherto associated almost exclusively with reinforced concrete.

Ac.Ca. building investigated flexible and adaptable over time prefabricated technological solutions, in order to overcome the formal, constructive and dimensional standardization of the components, which typically accompanied the developments of prefabricated constructions. Advanced modeling tools and methods capable of simulating multiple configurations that use this construction systems can support the development of flexible technological components that are compatible with different formal and functional combinations. This allows to understand the instances of mass-customization processes without affecting the performance profile of the components themselves.

Along with this technological flexibility, Ac.Ca. building offers a variety of formal solution that characterize the aesthetics of the architetural envelope. These solutions are linked to a customization that does not depend only on aesthetics but is aware of a diversified demand in terms of use of housing, transformability and cost control without ever compromising the architectural quality of the buildings being designed. These aspects are among the most difficult to satisfy with the use of prefabricated building systems.
TECHNOLOGICAL CHARACTERISTICS OF AC.CA. BUILDING

Ac.Ca. Building's technological system is composed of a steel structure combined with dry construction elements. The $6.600 \mathrm{~mm}$ structural span (divided into $11 \times 600 \mathrm{~mm}$ modules) allows the configuration of extremely flexible spaces, which can be combined in various planimetric configuration.

Beam and column are linked through rigid joint that allow to not use vertical and horizontal bracing. The structural joints are welded in plant workshop in order to create perfect connection. In the construction site, the beam-pillar connection are completed with fully restored bolted joints located slightly shifted to the actual connection. This led to the use of head-to-head connections that are more agile to be made in the construction site compared to welding and easier to dismantle at the end of the building's life. For the slabs a bidirectional framing was chosen: this allowed the elimination of floor bracing and a more convenient handling and trasport system. For a faster assembly, the slab of each structural bay is divided into 3 two-dimensional modules.

For the building envelope, hemp sandwich panels was designed from scratch. They are composed of two rigid hemp-wood panels that cover a hemp-wool core. This insulating material is contained within metal frames that allow anchoring to the bearing structure by bolting. All internal partitions are dry walls in hemp-wood panels mounted on a metal frame. For the bathrooms and kitchens panels of plastic laminate are used. Internal slabs are also composed with a dry layering on a thickness of 400 millimeters including a false ceiling used for the plant systems. Piping have been completly arranged in the cavities of internal partition and in the false ceiling. A further piping cavities is found in internal face of the building envelope. The absence of plant under the pavement avoids interrupting the continuity of the panels that compose a dry screed.

\section{APPROACHES TO SPATIAL LAYOUT OPTIMIZATION}

To understand the principles of research in the generation of spatial layouts it is necessary to address not only the architectural problem, but also its mathematical implications. The way in which we intend to solve these problems, which are mathematically often referred to as Constraint Satisfaction Problems (Graf \& Hower, 1996), greatly influence the manner in which a valid solution is generated. For example, if you opt for an evolutionary solving approach, it becomes appropriate to use generative processes that are intrinsically able to explore the design space on a performative basis.

Several approaches to layout generation are presented in this paper because. Thanks to the greater computational power available today, it is possible to use generative processes whose use in the past was reserved for the resolution of small problems. The selection of the proposed works was made by seeking historical relevance and the maximum heterogeneity of generative process. 
SPATIAL LAYOUT GENERATION PRE-

\section{EVOLUTIONARY ALGORITHM: CASE STUDIES}

This document briefly present researches on the generation of spatial layouts without using an evolutionary approach. Several of the presented researches are however suitable for the implementation of EMOA as they present methodologies for evaluating the efficiency of a solution (Ligget, 1985).

A large group of early research were characterized by the use of Operations Research to optimize the costs associated with the distribution aspects, while others do not address the problem of optimization but stop at satisfying sets of constraints and relationships.

The first experiments that proposed the automatic generation of solutions to architectural problems were, in fact, considered "immoral" as they would have risked generating unemployment among professionals (Negroponte, 1970, p. 39), despite them were based on a randomized "trial and error" procedure. Eric Teicholz in 1969 with GRASP (Generation of Random Access Site Plans) was able to generate layouts using predefined architectural modules with a randomized approach, managing to select the most promising solutions (Menges \& Ahlquist, 2011, p. 12). RUMOR was similar in its functioning and it was used for the randomized generation of housing plans. Its evolution, ALOKAT, allowed a hierarchization of the constraints applied to spatial layouts (Bernholtz \& Fosburg, 1972). Eastman (1971) with General Space Planner (GSP) not only proposes a layout generation method, but an entire computer augmentation system to simplify repetitive tasks that are faced every day in a design firm. GSP is a set of components for the design and resolution of constraint based problems, in which the spatial units had to respect bonds of adjacency, distance, sight, access and orientation. Further approaches to the generation of spatial layouts can be found in the catalog of the first exhibition of the Computer Art Society (1969)

\section{SPATIAL LAYOUT GENERATION WITH- EVOLUTIONARY ALGORITHM: CASE STUDIES}

The evolutionary approach places particular emphasis on the iterativeness of the generative process. The Reptile system (Frazer, 1974), although not specifically aimed at generating spatial layouts, represents a first attempt to produce parts of buildings by assembling coded spatial units. From this experimentation Frazes derives two principles common to generative systems (1995): conceptualize architectural information in one minimum coded configuration and describe the detail information in a subsequent output phase. Subsequent spatial layout generation methods, in particular the one proposed in this paper, are still based on the principle of minimizing the information necessary to produce a valid architectural configuration.

A framework that from its initial stages is aimed at the use of evolutionary algorithms is presented by Jagielski and Gero (1996). They address the formulation of a spatial layout as a quadratic assignment problem: given a set of $n$ distinct sites, $m$ distinct objects can be placed uniquely in the sites. Matematically, this approach defines a one-toone mapping optmization. Jagielski and Gero's experimentation involved the generation of office layouts.
Their framework provided for a hybrid approach, allowing the definition of some intended spatial units and reserving the evolutionary approach to a second part of the layout. The final output were optimized in respect to cost function. The whole problem was defined by a matrix-type approach, which showed important limitations in the post-processing phase of selection solution. In this research we can recognize important contributions on the mathematical aspects of space layout problems, such as their recognition in the class of nondeterministic polynomial-time hardproblem (NP-hard) and the intuition that a evolutionary approach is research method for optimal solutions also suitable for large and complex problems.

Subsequent research (Damski \& Gero, 1997) was based on the generation of spatial layouts using halfplanes: planes divided by an abstract border into two non overlapping areas. They shown, developing a dimensionless case study with halfplane with straight and parallel edges, how an evolutionary approach can generate admissible spatial solution with respect to a set of topological and directional constraint. Of particular interest is the type of output proposed: the developed codes do not generate a single admissible layout, but a family of possible topologies (different combinations of halfplans) from which the layouts can be generated. This hybrid approach greatly shifts the decision-making burden towards the designer.

Following this first group of research, the characteristics common to different approaches for the generation of spatial layouts were identified. Ligget acknowledge three main evaluation processes on the generated layouts (2000): mono-objective regarding the reduction of costs or paths lenghts; multi-objective regarding border conditions and overall characteristics; on the assessment of the adjiacency conditions of each spatial units. Elezkurtaj and Franck (2002) identify three characteristics for an optimal generative layout system: the presence of an interface able to modify dimensional and relational aspects of the individual spatial units as well as of the entire building; the possibility to modify a single spatial unit's preferred shape, orientation and adjacency; the possibility to modify the optimization process by making changes both on the spatial units and on the fitness functions during the analysis. These features enhance the post-processing of the proposed solutions, allowing more efficient and aware choices. In addition to these methodological progress, Elezkurtaj and Franck propose to approach the generation of spatial layout as bin-packing, nesting or cutting problem. It is a generation method which requires much time to obtain a valid solution without the use of an evolutionary approach.

An even different approach is given by the use of k-d trees (Knecht \& König, 2010). They are methods of multidimensional representation of set of points. The proposed methodology uses the k-d trees algorithms to divide a rectangular area by optimizing dimensions, ratios and topology. This research puts particular emphasis on the interaction between the generative mechanism and the designer, allowing him to directly influence the optimization process: it is possible to increase, decrease and change the weights and relations of the spatial unit's representative points during the analysis process. 


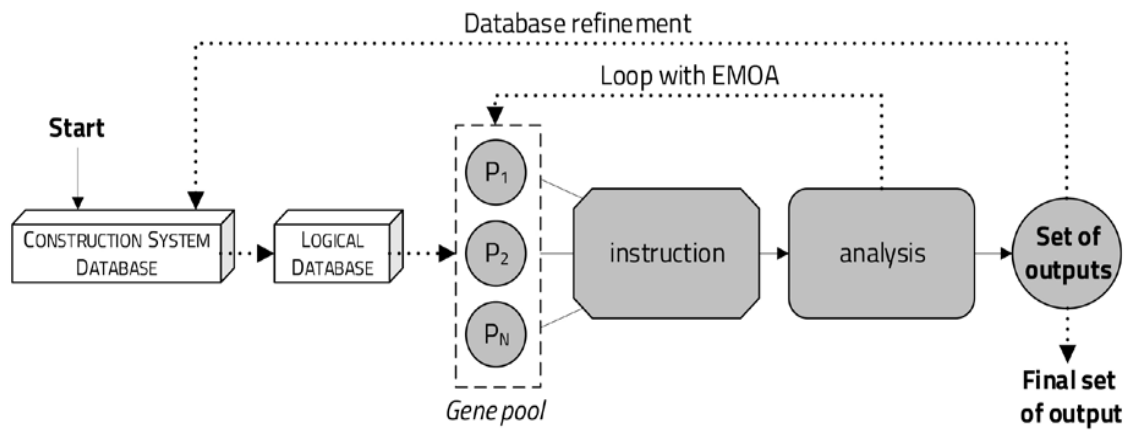

Figure 2: Proposed framework. In dark the typical steps of the parametric design process proposed by Tedeschi (2014). In white the implementations proposed by the authors to pursue the objectives of this paper.

More recent research propose the use a fuzzy modelling aimed at generating bubble diagrams in the concept design phase (Bayraktar \& Çağdaş, 2013) while others exceed some limits of previous research by offering greater attention to the connection spaces between each spatial units (Gravilov, Schneider, Dennemark, \& Koenig, 2020). Their final outputs also include components, such as Roomlstance and Houselnstance to use in the Grasshopper modeling environment. This last research has been applied to the design of public buildings' spatial layout where the weight of distribution spaces is particularly important.

Additional approaches that could be developed with an evolutionary approach are the Shape Grammar, based on the application of generative procedural rules and the Slice Tree Structures (Calixto \& Celani, 2015).

\section{METHODOLOGY}

The research presented in this paper minimizes the effort in the design of the different spatial units that compose the spatial layout. In fact, spatial units are treated as geometric shapes with minimal architectural and technological information. The main difference between past researches and the framework proposed in this paper lies in the greater effort in the design of spatial units which, due to their intrinsic characteristics, are suitable to generate spatial layout with a computational approach while maintaining a high level of architectural and technological definition

With reference to a typical schematization of the parametric approach to architectural design (Tedeschi, 2014), the authors propose to derive aggregative logics from an existing construction system thus building a logical database to be used for the generation of architectural spatial layouts. The framework is showed in Figure 2. The final output is linked to the states of $P_{x}$ variables trough the execution of a set of instruction which will be unfolded later. This approach allows to identify for each set of possibile spatial units that can be used a field of possible outputs, called design space, which itself needs to be designed in the meta-programming phase.

The analysis and optimization of the fitness function, which measure the performance of each outputs, are managed trought the use of EMOA. The final output, chosen through selecation criteria that will be unfolded later, will be compared with an architectural plan designed in other researches without the use of the framework proposed in this paper

\section{SOFTWARE AND TOOLS USED}

The research presented in this paper was conducted using primarily the Rhinoceros 3D modeling environment (Version 6.22; Robert McNell \& Associates, 2020). The spatial units' database was constructed using only brep and polyline as primitives. The computational approach is made possible by the use of the Grasshoppe visual programming software (Build 1.0.0007, Robert McNell \& Associates, 2020) contained in Rhinoceros 3D (from version 6).

Octopus (version 0.4) and Wallacei (version 2.55) were used for the implementation and analysis with EMOA. The first one was used in the problem setting's phase thanks to his flexibility features (Vierlinger \& Hofmann, 2013). In particular, the greater flexibility of Octopus in the visualization of the Pareto Surface and relatives phenotypes has allowed to identify errors in the defnition of the fitness funcion, improving the calculation times of optimal solutions. Wallacei (Makki, Showkatbakhsh, \& Song, 2019) was preferred in the final stages of the work because its ability to analyze the solutions postponing the generation of phenotypes makes it particularly agile in the selection phase. Based on NSGA-II algorithm, Wallacei puts emphasizes on non-dominated solution and uses an elitist principle while preserving diversity (Deb, 2011). The extensive analysis tools contained in Wallacei Analytics was used to identify the best design outputs on a scientific basis as showed later.

\section{CONSTRUCTION OF THE DATABASE}

The digitalization of Ac.Ca Building's existing architectural databases was done aiming to obtain minimum coded configuration able to be quickly analyzed and to be representative of the architectural configuration. During the assembly of spatial layout, movements and rotations on the coded spatial units are widely used, hence the need to minimize the information necessary to describe the architectural database. The reconstruction of architectural plants with higher levels of detail is postponed, thanks to the functioning of Wallacei, to the final selection phase.

A simplification was made on the modular coordination of Ac.Ca Building in order to use fitness function based on topological and geometrical checks. The computational 
model is based on an axial reference coordination system, unlike the original system that uses a bounduary reference coordination stestem. In the selection phase this simplification is eliminated so that the final outputs respect the rule of Ac.Ca. building construction system. In Figure 5 and Figure 7 we can see the computational and the construction models.

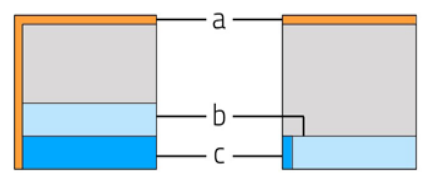

Figure 3: Information of the coded spatial units (CSU)

A typological submodel of Ac.Ca. Building, in the framework developed in this paper, is defined with only 3 types of information for its use in a logical database. With reference to Figure 3 a coded spatial unit (CSU) is composed of:

a. A polyline representing the position of the building envelope. It is essential because Ac.Ca. building uses a prefabricated envelope with different components.

b. A brep represeting the "services band". It allows to introduce fitness functions regarding rationalization and technological flexibility in complex volume.

c. A brep representative of "distribution band" to different housing. If a typological submodel has the entrance positioned directly on the border, a minimum sized brep has been introduced in the CSU to ensure the correct functioning of the fitness function subsequently unfolded.

CSU information need to be be minimized to improve computing performance. However, this simplification must still allow in a subsequent phase to reconstruct the architectural and technological information of the spatial layouts. It is possible to generate different CSU with a parametric approach. This would allow to satisfy the fitness functions with greater effectiveness for the design of more complex building than the benchmark case presented in this paper. Previous research, based on the use of very simple CSUs, has shown the possibility of adapting the logical database to the problem being faced (Frazer, 1995).

\section{PROBLEM SETTING}

The benchmark cases presented in this paper applies the proposed framework to the spatial layout design of a small tower building for residential uses as shown in Figure 4.

The plan has a square shape with each side consisting of 3 typological modules. The stairwell, occupying an entire module, is fixed in the center. The typological submodules that can populate this plan have already been designed in other researches and consist of small apartments for 1 or 2 people calibrated to the functional minimums of Italian residential regulations. The available database for this benchmark consist in two types of apartment with their variations regarding the position of the entrances and the building envelope.

This typological case has also been presented in other scientific works with different design methodologies and purposes (Lucente, 2020).

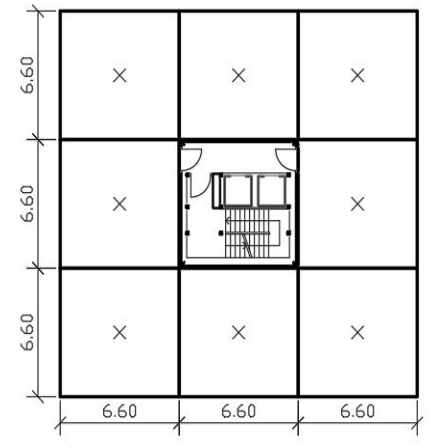

Figure 4: Spatial layout to be designed

\section{A "BRUTEFORCE" APPROACH TO SPATIAL LAYOUT GENERATION}

A progressive increase of the available computational power, greater ease of access to it in both economic and technical terms, as well as new forms of cloud-based computation, have allowed the computational approach to architectural design to permeate not only academic researches, but also the profession. In most cases, the generative approaches briefly described in this paper need to be simplified in the first phases of work in which the main objective is to validate a general framework, postponing the refinements of the generative process to subsequent phases. For these reasons, the generative approach used in this paper is easy to codify and manage.

The first operations concern the generation of the bounduary of the plan to be optimized. This is accomplished by identifying the cells that host the typological submodules in the form of polylines. The center of each cell is also generated to facilitate the subsequent translation and rotation operations of the typological submodules.

Afterwards the floor space is populated by assigning a typological submodule contained in the database, under the form of a coded spatial unit, to each plan module. For each module a parameter capable of assigning 4 different orthogonal rotation is also defined.

It is clear that, for the generation of more complex plans and volumes, the boundaryof the floor space can also become an aspect to be optimized concerning energy, structural, technological or other fitness functions.

The generative approach used in this research aims to minimize the effort of writing the algorithms and shift the computational burden to the EMOA.

After verifying the effectiveness of the translation of the Ac.Ca. Building database and the convergence of the EMOA outputs towards already known and valid solutions, it will be appropriate to refine the generative process by looking at possible approaches capable of significantly reducing computation times and improving the explorability of the design space. In particular, for the use of significantly larger housing modules, already present in the architectural database of the Ac.Ca Building, it will be necessary to rethink the assembly aspects of the spatial layout. 

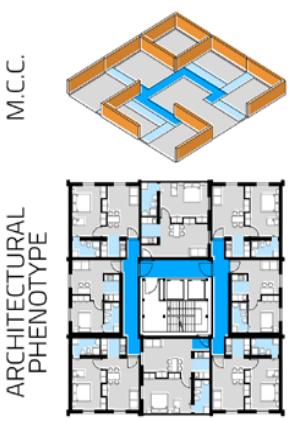

Gen. 11 Rank 1 on FF

Ind. $8 \quad \mathrm{FF}^{\mathrm{F}}: 41.0$
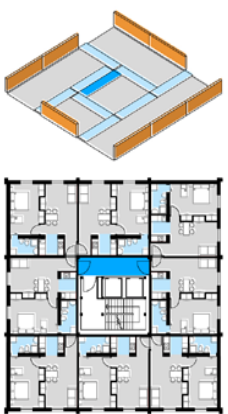

Gen. 41 Rank 1 on FF2

Ind. 1 FF2: 11.02
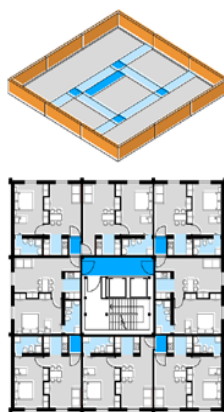

Gen. 60 Rank 1 on FF 1

Ind. $4 \quad F F 2: 19.4$

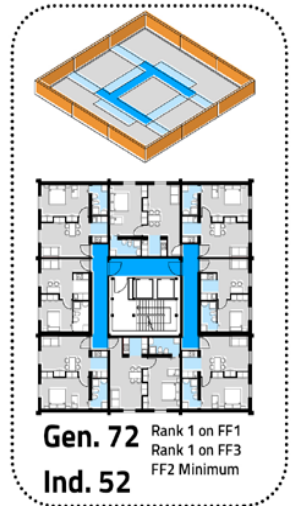

Ind. 52

Figure 5: Rank 1 phenotype for FF1, FF2 and FF3. The selected output is highlighted on the right.

\section{FITNESS FUNCTIONS}

When an analysis through EMOA is done it necessary to build fitness function that are a measure of the efficiency of the solutions generated. In this benchmark case was necessary to evaluate both topological and geometrical aspects. Among the topological constraints, concerning hierarchical relationships between spatial elements such as adjiacency and proximity, applied in this paper we can find:

d. Congruence between the external borders of the CSU and the building envelope

e. Adjacency between the apartment entrances and the distribution band

f. Number of surfaces composing the distribution system. Necessarily equal to one in the final output to guarantee accesses to each apartment.

g. Number of breps composing the service band. This is done forecasting the application of the proposed framework to multi-storey design where a rationalization of the technological system should be done.

To allow greater exploration of the design space, the algorithm can also generate solutions that do not respect the constraints previously explained. This degree of dissatisfaction is measured and is a task of the EMOA not preferring these solutions in breeding processes. The only geometric constraint, properly so called, concerns the minimization of the distribution surface's area. However, geometrically quantifiable aspects have been derived from various topological constraints, such as:

h. Length difference between the sum of the envelope of the coded spatial units and the perimeter of the floor plan to populate. Necessarily equal to 0 in the final solution.

i. Length of the envelope not falling on the perimeter of the floor plan to populate. Necessarily equal to 0 in the final solution.

The aspects $\mathbf{h}$ and $\mathbf{i}$ are apparently redundant but their simultaneous use avoids inadmissible situations such as those shown in Figure 6

Ultimately, three fitness functions have been derived from the previously described aspects:
FF1: Measurement of apartments accessibility.

FF2: Measurement of the area of the distribution system

FF3: Congruence of the building envelope
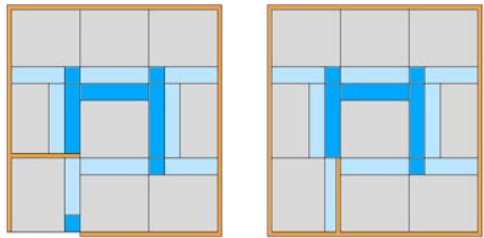

Figure 6: On the left an aggregation that doesn't minimize i, and on the right an aggregation that doesn't minimize $\mathbf{h}$.

\section{GENERATION OF ARCHITECTURAL FENOTYPE}

The most promising outputs are selcted in the selection phase. The proposed framework associate to each promising solution a phenotype characterized by a high level of architectural definition. This is done to facilitate the decision-making process. To do this a parallel database that associate to each coded spatial units an architectural phenotype is created. In this parallel database we can find most of the information of the Ac.Ca. Building system as shown in Figure 7.
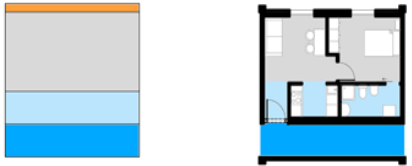

Figure 7: On the left an example of a coded spatial units and on the right his architectural phenotype containing information of the Ac.Ca. Building System.

The outputs presented in Figure $\mathbf{5}$ were built by automatically recreating an architectural plan containing information such as the building envelope, the furniture and partition systems 


\section{OUTPUT SELECTION AND FRAMEWORK VALIDATION}

From the analysis previously exaplained on the fitness functions used, it is clear the need to select outputs that have values of the fitness function FF1 regarding the building envelope equal to 0 and FF2 regarding the distribution system equal to 1 . In the selection phase, among these two constraints, are chosen solutions that minimize the fitness function FF3 regarding the area of the distribution system. These combinations of values of the fitness functions ensure optimized and architecturally admissible outputs. The location of these architecturally most promising solutions in the parallel coordinate plot are shown in Figure 8.

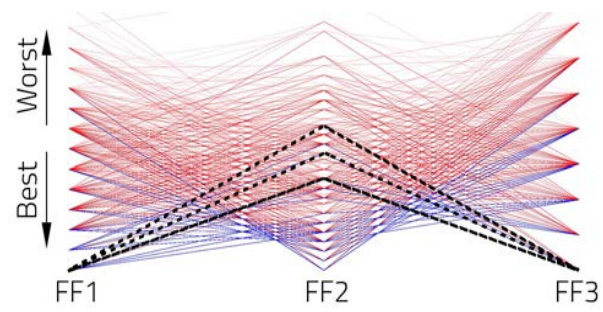

Figure 8: The parallel coordinate plot of the fitness function values. Solutions that meets the previously explained constraints are highlighted in dark. In red are shown the values of the solution produced in the first generations while in blue in the most recent generations.

In Figure 5 three solutions of Rank 1, with respect to FF1, FF2 and FF2, are shown on the left. However, these solutions are not architecturally admissible mainly due to the incorrect position of the bulding envelope and the inability to access all apartments. The selected output, on the right, on the other hand, is a solution that minimizes FF2 and simultaneously impose the Rank 1 value on FF1 and

FF3.

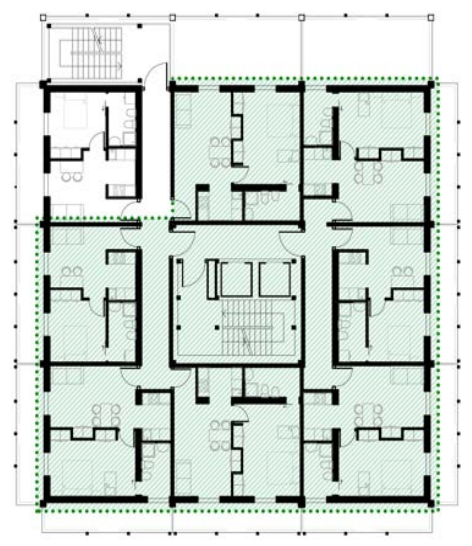

Figure 9: Difference between the solution selected through EMOA and an architectural plan designed without a generative approach. The congruent parts are highlighted in green.

The selected solution if superimposed with a layout designed without a generative approach (Lucente, 2020) has approximately $89 \%$ congruence, as shown in Figure 9. The differences depend on the missing implementation of an emergency exit for fire protection. It will be possible to implement this aspect in future works by creating a fitness function that rewards spatial layouts in which the distribution system touches one time the perimeter of the spatial layout.

\section{FURTHER WORKS}

Further research will be aimed at improving the efficiency of the generative algorithm and the introduction of fitness functions capable of considering aspects related to sustainability and spatial flexibility. Subsequent benchmark cases will concern more complex plan geometries also introducing the theme of multistory building as well as larger submodules, already present in the Ac.Ca building database. The authors are convinced that the actual benefits of the proposed method increses with the complexity of the layout and volumes being designed.
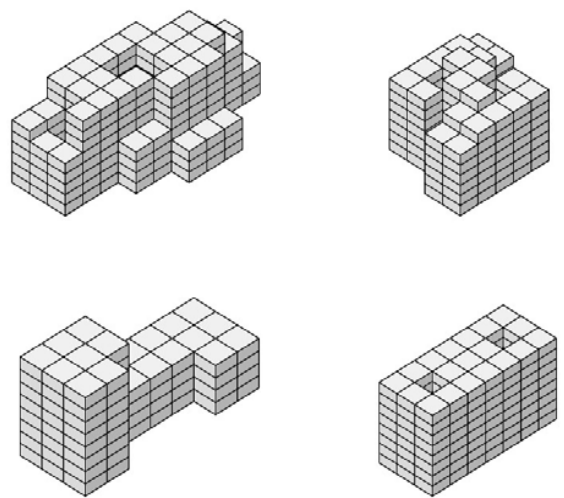

Figure 10: Complex volumes to which apply the proposed method in future research.

In the phenotype construction phase, is possible to automate the creation of Building Information Models (BIM) of the most promising spatial layouts. This could improve the interdisciplinarity of the proposed framework and overcome some lack of interoperability with the creation of information interchange models, like Industry Foundation Classes (IFC). These furtherworks are made possibile by the high level of architectural and technological detail of the Ac.Ca. Building system database.

\section{CONCLUSIONS}

The methodological framework shown in Figure 2 can be extended to multistory design using the Ac.Ca building system and, more generally, to the design of significant parts of the building, such as the architectural envelope, with a computational approach using prefabricated technologies.

Unfolding the computational logic that could be used with a specific construction system requires a deep knowledge of the system itself. Following this effort, however, it is possible to explore the design space in a significantly 
greater way by linking aspects of technology, economic, structure, energy and other fields to the architectural design. As shown in this paper, the proposed framework is applicable both in the predesign phase with the aim of evaluating the application of a construction system, but also in the design development phase, being able to generate highly defined phenotypes, ready to use in a BIM environment. This paper shows that even traditional design methods can benefit from parametric design.

The use of EMOA shifts the emphasis from the design of a single output to the design of a set of instruction capable of generating a great number of outputs. Another shift is from the design of a single output to the selection of the most promising output. Selection is a fundamental aspect in the use of EMOA, and despite the existence of analysis techniques on Pareto surface or even the use of machine learning to cluster promising outputs, a selection based on Rank analysis as done in this paper remains a more manageable decision making process.

\section{ACKNOWLEDGMENT}

The Ac.Ca. building construction system was developed in a funded research in which all the authors participated under the scientific supervision of Roberta Lucente. . The architectural system was mostly designed by Roberta Lucente and Giuseppe Canestrino while the technological system by Laura Greco and Francesco Spada in a spirit of cooperation and integration of different knowledge. The innovative research presented in this paper, including the development of the proposed method, the visual algorithms for spatial layout design and the study of similar research was done by Giuseppe Canestrino.

\section{REFERENCES}

Bayraktar, M., \& Çağdaş, G. (2013). Fuzzy layout planner. A simple layout planning tool for early stages of design. Computation and Performance - Proceedings of the 31st eCAADe Conference (p. 375-382). Delft: Sevil.

Beck, K., Beedle, M., Bennekum, A., Cockburn, A., Cunningham, W., Flowler, M., Thomas, D. (2001). Manifesto For Agile Software Development. Tratto il giorno May 272020 da agilemanifesto.org

Bernholtz, A., \& Fosburg, S. (1972). Spatial allocation in design and planing. DAC 172: Proceedings of the 9th Design Automation Workshop, (p. 181-189).

Calixto, V., \& Celani, G. (2015). A literature review for space planning optimization using an evolutionary algorithm approach: 1992-2014. Proceedings of the 19th Conference of the Iberoamerican Society of Digital Graphics - vol. 2 - ISBN: 978-85-8039-133-6, (p. 662-671). Florianópolis.
Computer Arts Society. (1969). Event One Catalogue. Tratto il giorno May 26, 2020 da https://computer-artssociety.com/uploads/event-one-1969.pdf

Damski, J., \& Gero, J. (1997). An evolutionary approach to generating constraint-based space layout topologies. CAAD Futures (p. 855-874). Dordrecht: Kluwer Academic Publishing.

Deb, K. (2011). Multi-objective optimization using evolutionary algorithms: an introduction. Kanpur: KanGAL Report.

Eastman, C. (1971). GPS: A system for computer assisted space planning. DAC '71: Proceedings of the 8th Design Automation Worshop, (p. 208-220)

Elezkurtaj, T., \& Franck, G. (2002). Algorithmic support of creative architectural design. Umbau 19, 129-137.

Frazer, J. (1974, April). Reptiles. Architectural Design, p. 231-239.

Frazer, J. (1995). An evolutionary Architecture. London: Architectural Association.

Graf, W. H., \& Hower, W. (1996). A bibliographical survey of constraint-based approaches to CAD, graphics, layout, visualization, and related topics. Knowledge-Based Systems(9), p. 449-464.

Gravilov, E., Schneider, S., Dennemark, M., \& Koenig, R. (2020). Computer-aided approach to public buildings floor plan generation. Magnetizing floor plan generator. Procedia Manufacturing 44 (p. 132-139). Elsevier.

Jagielski, R., \& Gero, J. S. (1996). A genetic programming approach to the space layout planning problem. CAAD Futures, 875-884.

Knecht, K., \& König, R. (2010). Generating floor plan layouts with k-d trees and evolutionary algorithms. XIII Generative Art Conference (p. 238-253). Milano: Domus Argenia.

Ligget, R. (1985). Optimal spatial arrangment as a quadratic assignment problem. Design Optimization, 1-40.

Liggett, R. S. (2000). Automated facilities layout: past, present and future. Automation in Construction(9), p. 197-215.

Lucente, R. (2020). Abitare Acciaio. Siracusa: LetteraVentidue.

Makki, M., Showkatbakhsh, M., \& Song, Y. (2019). Wallacei Primer 2.0. [Online]. Tratto da www.wallacei.com

Menges, A., \& Ahlquist, S. (2011). Computational Design Thinking. Chichester: John Wiley \& Sons.

Negroponte, N. (1970). The architecture machine. Toward a more human environment. Cambridge: The MIT Press.

Tedeschi, A. (2014). AAD_Algorithms-Aided Design. Edizioni Le Penseur.

Vierlinger, R., \& Hofmann, A. (2013). A framework for flexible search and optimization in parametric design. Design Modelling Symposium - Rethinking Prototyping , (p. 1-9). Berlin.

Williams, J. H. (2008). Employee engagement: Improving articipation in safety. Professional Safety, , 12(53), 40-45. 